\title{
LIMITS ON JUMP INVERSION FOR STRONG REDUCIBILITIES
}

\author{
BARBARA F. CSIMA, ROD DOWNEY, AND KENG MENG NG
}

\begin{abstract}
We show that Sacks' and Shoenfield's analogs of jump inversion fail for both tt- and wtt-reducibilities in a strong way. In particular we show that there is a $\Delta_{2}^{0}$ set $B>_{t t} \emptyset^{\prime}$ such that there is no c.e. set $A$ with $A^{\prime} \equiv_{w t t} B$. We also show that there is a $\Sigma_{2}^{0}$ set $C>_{t t} \emptyset^{\prime}$ such that there is no $\Delta_{2}^{0}$ set $D$ with $D^{\prime} \equiv_{w t t} C$.
\end{abstract}

\section{INTRODUCTION}

The concern of this paper is the interaction of two basic notions from computability theory. These are the jump operator and reducibilities stronger than Turing reducibility which are of the tabular type. We anwer a question of Anderson [And08] by showing that there are no analogs of Sacks Jump Inversion Theorem [Sac63] and Shoenfield's Jump Inversion Theorem [Sho59] for these strong reducibilties.

The study of strong reducibilities has been part of computability since the dawn of the subject, as witnessed by Post's paper [Pos44]. $A$ is Turing reducible to $B$, $A \leq_{T} B$, means that $A$ can be computed by $B$ via any oracle access mechanism. It is clearly natural to ask what happens when we restrict the access mechanism in the reduction from $A$ to $B$. Tabular reducibilies such as weak truth table (wtt-) and truth table (tt-) reduciblities do not allow the reduciblity to be adaptive. Thus, as is well known, $A \leq_{t t} B$, is defined as $x \in A$ iff $B \models \sigma_{f(x)}$ where $f$ is a computable function and $\sigma_{f(x)}$ is the $f(x)^{\text {th }}$ truth table. As is also well known a truth table reduction is simply a Turing reduction $\Phi$ which is total for all oracles. Weak truth table reducibility simply has the truth table being partial, or $\Phi^{B}=A$ where the use of the computation $\varphi(x)$ is a computable function. Thus in either case we are not allowed to adapt the size of the reduction as the oracle $B$ varies. $A \leq_{t t} B$ implies $A \leq_{w t t} B$ but it is easy to construct examples where the converse fails.

These reducibilities also arise very naturally when we consider reducibilities coming from reductions in mathematical structures. For example, the reduction of the word problem to the conjucacy problem in combinatorial group theory is a ttreduction and the degrees of bases of c.e. vector spaces are naturally represented by weak truth table degrees (Downey and Remmel [DR89]).

In recent times, truth table reducibility has become a central area of interest as it has been shown to be a natural reducibility to study in algorithmic randomness, a fact first realized by Demuth [Dem88]. The point here is that if $A \leq_{t t} B$, via $\Phi^{B}=A$, with $\Phi$ total on all oracles, then we can use $\Phi$ to translate between measures effectively. For instance if $B$ is random with respect to uniform measure, and $A$ is noncomputable, $A$ will be random wih respect to the measure generated by the inverse of $\Phi$. Thus, for instance, truth table degrees are absolutely central

B. Csima was partially supported by Canadian NSERC Discovery Grant 312501.

The second and third authors were partially supported by the Marsden Fund of New Zealand. 
to the deep investigations of Reimann and Slaman [RS08a, RS08b] on sets never continuously random. They are also deeply connected with things like the CantorBendixson rank of sets for a similar reason.

All of this recent work has highlighted our lack of understanding as to how the finer structure of the $(\mathrm{w}) \mathrm{tt}$-degrees relates to the jump operator. The halting problem is a fundamental object of computability theory, and the jump $A^{\prime}=\{e$ : $\left.\Phi_{e}^{A}(e) \downarrow\right\}$ is the relativized form of the halting problem.

For Turing reducibility, we know a lot about how the jump operator behaves. The most basic theorem is Friedberg's Jump Inversion Theorem [Fri58], that if $X \geq_{T} \emptyset^{\prime}$ then there is a set $A$ with $A^{\prime} \equiv_{T} X \equiv_{T} A \oplus \emptyset^{\prime}$. Early on, Mohrherr [Moh84] proved that if $X \geq_{t t} \emptyset^{\prime}$, then there is a set $A$ with $A^{\prime} \equiv_{t t} X$. Mohrherr's proof came from an analysis of Friedberg's Theorem, and resulted in a 1-generic set $A$. It was only much later that Anderson [And08] proved that indeed the full analog of Friedberg's Theorem held; if $X \geq_{t t} \emptyset^{\prime}$ then there is a set $A$ with $A^{\prime} \equiv_{t t} X \equiv_{t t} A \oplus \emptyset^{\prime}$. Anderson's theorem was more difficult than Mohrherr's, and the method employed by Friedberg (which will give generics sets) provably fails, so that arguments akin to those from information theory were necessary.

All of this lead to the present paper. The most important sets in computability theory are the c.e. sets as well as those computable from the halting problem, the $\Delta_{2}^{0}$ sets. Shoenfield [Sho59] had proven a jump theorem for such sets. Namely for any $\Sigma_{2}^{0}$ set $X \geq_{T} \emptyset^{\prime}$ there is a $\Delta_{2}^{0}$ set $A$ with $A^{\prime} \equiv_{T} X$. Famously, Sacks used the infinite injury method to show that the same result held with $A$ a computably enumerable set, and after that many other intricate jump theorems were found culminating in Robinson's Jump Interpolation Theorem [Rob71]. (See Soare [Soa87] for more details.)

Anderson asked: do the analogs of any of these basic theorems hold for tt- or perhaps wtt-reducibilities? We prove that the analogs fail to hold and in fact that they fail in more or less the strongest way that they can. Our first result shows that Sacks' Jump Inversion Theorem fails for both the tt- and wtt-reducibilities, by constructing a $\Delta_{2}^{0}$ counter-example. We will in fact prove something stronger:

Theorem 3.3. For any computable sequence of $\Delta_{2}^{0}$ sets $\left\{V_{e}\right\}_{e \in \mathbb{N}}$ (given by their $\Delta_{2}^{0}$ indices), there exists a $\Delta_{2}^{0}$ set $S \geq_{t t} \emptyset^{\prime}$ such that for every $e, V_{e}^{\prime} \neq_{w t t} S$.

From Theorem 3.3 we deduce the failure of Sacks' Jump Inversion for both ttand wtt-reducibilities:

Theorem 3.4. There exists an $\omega+1$-c.e. set $S>_{t t} \emptyset^{\prime}$ such that there is no c.e. set $A$ with $A^{\prime} \equiv_{w t t} S$.

Hence $S$ is in the first place of the Ershov Hierarchy where a counter-example can be. The result also give an interesting fact about the $\Delta_{2}^{0}$ wtt-degrees which are realized by the jump of low c.e. sets. Clearly there are such wtt-degrees $\mathbf{a}>\mathbf{0}^{\prime}{ }_{w t t}$, namely the wtt-degrees of the jump of low but not superlow c.e. sets. Our result shows that not every wtt-degree $\mathbf{a}>\mathbf{0}^{\prime}$ wtt can be realized by the jump of a (low) c.e. set.

Our second result shows that the analogue of Shoenfield's Jump Inversion Theorem fails for both the tt- and wtt-reducibilities. By Mohrherr's result, the counterexample $S$ has to be strictly $\Sigma_{2}^{0}$ :

Theorem 3.5. There exists a $\Sigma_{2}^{0}$ set $S>_{t t} \emptyset^{\prime}$ such that there is no $\Delta_{2}^{0}$ set $A$ with $A^{\prime} \equiv_{w t t} S$. 
1.1. Notation. We follow standard notation for Computability Theory, as found in Cooper [Coo04] and Soare [Soa87].

\section{The BASIC MOdUle}

2.1. The plan for the c.e. case. Let $\left(\Gamma_{e}, \Delta_{e}, \gamma_{e}, \delta_{e}\right)_{e \in \omega}$ run through all possible 4-tuples where $\Gamma_{e}$ and $\Delta_{e}$ are Turing functionals, and $\gamma_{e}$ and $\delta_{e}$ are partial computable functions. Let us suppose we wanted to prove Theorem 3.4 directly by constructing $S$. We must then meet for all $e \in \omega$ the requirements:

$$
R_{e}: \Gamma_{e}^{V_{e}^{\prime}} \neq S \text { or } \Delta_{e}^{S \oplus \emptyset^{\prime}} \neq V_{e}^{\prime},
$$

where $V_{e}$ is the $e^{t h}$ c.e. set, and $\gamma_{e}$ and $\delta_{e}$ bound the uses of the computations of $\Gamma_{e}$ and $\Delta_{e}$, respectively. Then $S \oplus \emptyset^{\prime}$ will be the desired set. Note that the requirements automatically ensure that $S \oplus \emptyset^{\prime} \not \equiv_{w t t} \emptyset^{\prime}$.

Suppose we wanted to satisfy $R_{e}$. We can first try making $\Delta^{S \oplus \emptyset^{\prime}} \neq V^{\prime}$ (for the purpose of the discussion we drop subscript $e$ ). In particular we assume that the recursion theorem gives us infinitely many indices $x_{1}, x_{2}, \cdots$ for which we can control $V^{\prime}\left(x_{i}\right)$. The obvious plan is to keep $V^{\prime}\left(x_{1}\right)=0$ until $\Delta^{S \oplus \emptyset^{\prime}}\left(x_{1}\right) \downarrow=0$. We then make $V^{\prime}\left(x_{1}\right)=1$ by enumerating an axiom with some use $V\lceil u$. The only way in which $\Delta^{S \oplus \emptyset^{\prime}}\left(x_{1}\right)$ can later change to be 1 , is for some number $<\delta\left(x_{1}\right)$ to enter $\emptyset^{\prime}$. Our next step would be then to extract $x_{1}$ out from $V^{\prime}$; if we could always do this then we would know what to do. We would alternate the value of $V^{\prime}\left(x_{1}\right)$, and we will eventually succeed because $\emptyset^{\prime}$ is c.e. and the use $\delta\left(x_{1}\right)$ is fixed. Unfortunately we only have partial control over $V^{\prime}$ and extraction can only be achieved by forcing a change in $V\lceil u$.

We can start another line of attack by trying to make $\Gamma^{V^{\prime}} \neq S$ true. We pick an attacker $z$ for $S$, and for simplicity let us first consider the case where $\Gamma$ is an $m$-reduction; i.e. $z \in S$ iff $q \in V^{\prime}$ for some $q$. We begin by making $S(z)=1$, and wait for $V^{\prime}(q)=1$, i.e. $\Phi_{q}^{V}(q) \downarrow$ with some use $u$. Note that while the uses on $\Delta$ and $\Gamma$ are bounded, this use $u$ may be unbounded. We then begin the attack above by enumerating an axiom $\Phi_{x_{1}}^{V}\left(x_{1}\right)$ with the same use $u$, and wait for a $\emptyset^{\prime}$-change.

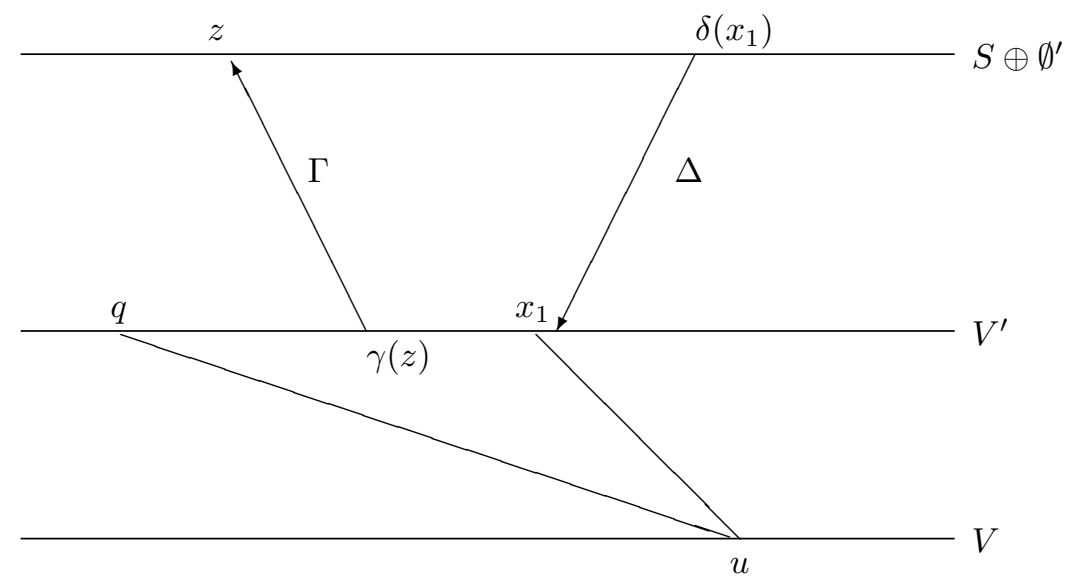

If no $\emptyset^{\prime}$-change occurs then it is clear that we would succeed at $\Delta^{S \oplus \emptyset^{\prime}}\left(x_{1}\right) \neq$ $V^{\prime}\left(x_{1}\right)$. If on the other hand a $V\left\lceil u\right.$ change occurs before a $\emptyset^{\prime}$-change, then we would wait for $\Phi_{q}^{V}(q) \downarrow$ again with a new use $u^{\prime}$, and then make $\Phi_{x_{1}}^{V}\left(x_{1}\right)$ converge 
with the same use $u^{\prime}$. The point is that if $V$ changes infinitely often this way with no $\emptyset^{\prime}$-change, then $V^{\prime}(q)=0$ and we would succeed via $\Gamma^{V^{\prime}}\left(z_{e}\right) \neq S(z)$. Lastly if $\emptyset^{\prime}$ changes then we would remove $z$ from $S$, and wait for $\Phi_{q}^{V}(q)$ to become undefined again. This has to happen (unless already $\Gamma^{V^{\prime}} \neq S$ ), and so at some point we will also get a clear on $\Phi_{x_{1}}^{V}\left(x_{1}\right) \uparrow$. We can then repeat by making $S(z)=1$ again. Note that we only toggle $z$ in $S$ whenever $\emptyset^{\prime}$ changes below $\delta\left(x_{1}\right)$, so requirement $R_{e}$ can be satisfied with only finite action on $S$ (although we might possibly enumerate infinitely many axioms for $x_{1}$ ).

The strategy for a general wtt-reduction $\Gamma$ is as above, but we will run a separate copy of the strategy above for each possible configuration of the use $V_{e}^{\prime}\left\lceil\gamma_{e}\left(z_{e}\right)\right.$. We have $2^{\gamma_{e}\left(z_{e}\right)}$ many different $x$ 's corresponding to each different configuration of $V_{e}^{\prime}\left\lceil\gamma_{e}\left(z_{e}\right)\right.$. At each stage we look at the current approximation for $V_{e}^{\prime}\left\lceil\gamma_{e}\left(z_{e}\right)\right.$ (see section 2.2) and apply the above plan. As in the basic case we will only toggle $z_{e}$ in $S$ if $\emptyset^{\prime}$ changes below some $\delta_{e}$-use. Each time we toggle $z_{e}$ we will force the configuration $V_{e}^{\prime}\left\lceil\gamma_{e}\left(z_{e}\right)\right.$ to change. This can only move lexicographically right finitely many times (consecutively), hence after finitely much toggling of $z_{e}$, the configuration for $V_{e}^{\prime} \backslash \gamma_{e}\left(z_{e}\right)$ will return to an earlier one. This makes all the $x_{\tau}$ (for all the $\tau$ on the right of the current $V_{e}^{\prime}$-configuration) undefined, so that if $\tau \subset V_{e}^{\prime}[s]$ holds again later we can use $x_{\tau}$ to cause further $\emptyset^{\prime}$-changes.

The above works when diagonalizing against all c.e. sets. However if $V$ is $\Delta_{2}^{0}$ then whenever the configuration of $V^{\prime} \uparrow \gamma_{e}\left(z_{e}\right)$ returns to an earlier one, there is no guarantee that all $x_{\tau}$ (for $\tau$ on the right of the current $V^{\prime}$-configuration) become undefined. However we can show that some amount of progress has been made because in this case, $V$ has to return to a previous $x_{\tau}$ axiom, and thus we will threaten $V$ to be not $\Delta_{2}^{0}$.

From the above discussion, the reader will notice that the different requirements act almost independently of one another. In fact all that a single requirement needs to know is the correct initial segment of $S$. When diagonalizing against all $\Delta_{2}^{0}$ sets, it may be possible for a requirement to flip $S$ infinitely often. However we do not need to re-pick the followers of lower priority requirements due to this reason. The only reason why $R_{2}$ needs to pick a new $z_{2}$ is because $R_{1}$ has seen $\delta_{1}, \gamma_{1}$ converge, and wants to protect now a certain segment of $S$. This initialization happens only finitely often (despite $R_{1}$ flipping $S\left(z_{1}\right)$ infinitely often). Therefore it will be straightforward to combine the requirements, and will not require a tree argument as one usually expects in full approximation arguments.

2.2. The modular approach. We proceed in a general setting, and then obtain the main theorems as corollaries. We start by fixing a computable sequence $\left\{V_{e}\right\}_{e \in \mathbb{N}}$ of possible $\Delta_{2}^{0}$-approximations. That is, $V_{e, s}(x)$ is a computable function of $e, s, x$. We say that $V_{e}$ is $\Delta_{2}^{0}$ if $\lim _{s} V_{e, s}(x)$ exists for all $x$ and $V_{e}(x)$ is this limit.

Let the natural approximation of the jump of $V_{e}$ be $V_{e, s}^{\prime}(n)=1$ iff $\Phi_{n, s}^{V_{e, s}}(n) \downarrow$ (as is customary we assume the hat trick, that there must be a divergence between consecutive convergences with different uses, see Soare [Soa87]). If $V_{e}$ is $\Delta_{2}^{0}$ then this serves as a natural $\Sigma_{2}^{0}$-approximation to the characteristic function of $V_{e}^{\prime}$ in the sense that $V_{e}^{\prime}(n)=\liminf _{s} V_{e, s}^{\prime}(n)$ for every $n$. However when approximating $V_{e}^{\prime} \uparrow x$ as a finite string, $V_{e, s}^{\prime}\left\lceil x\right.$ is obviously not ideal because $V_{e}^{\prime} \uparrow x$ might not be the lexicographically leftmost string specified by $V_{e, s}^{\prime}\lceil x$ at infinitely many $s$. It is easy 
to fix this by delaying any entry of $n$ into the (approximation for the) jump by the following.

We define another approximation $Q_{e}\left\lceil x[s]\right.$ for $V_{e}^{\prime}\lceil x$ this time by induction as follows: $0 \in Q_{e}[s]$ iff $\Phi_{0, s}^{V_{e, s}}(0) \downarrow$. For $n>0$, let $t<s$ be maximal such that $Q_{e}\left\lceil n[t]=Q_{e}\left\lceil n[s]\right.\right.$. If $\Phi_{n, r}^{V_{e}, r}(n) \downarrow$ for all $t<r \leq s$, and $V_{e}$ has been stable up till the use during this period, declare $n \in Q_{e}[s]$, and declare $n \notin Q_{e}[s]$ otherwise. Hence if $V_{e}$ is $\Delta_{2}^{0}$ then the lexicographically leftmost segment $Q_{e}\lceil x[s]$ specified infinitely often is the segment of the true jump $V_{e}^{\prime} \uparrow x$. The "delayed" approximation $\left\{Q_{e}[s]\right\}_{s}$ will be used when deciding whether or not to act for a module, since it is correct infinitely often. Furthermore the delayed approximation $Q_{e}$ for $V_{e}^{\prime}$ is obtained effectively in $e$.

We have infinitely many modules $M_{\theta, e}$ indexed by a finite binary string $\theta$ and $e \in \mathbb{N}$. Here $M_{\theta, e}$ works in a similar way as requirement $R_{e}$ above, and guesses that $\theta \subset S$. It outputs (effectively) an infinite binary sequence $m_{\theta, e}$ listing the stage by stage guesses as to whether our toggle point $z_{\theta, e}$ is in $S$, as well as a number $d_{\theta, e}$ such that if $V_{e}$ is $\Delta_{2}^{0}$, then

(P1) $m=\lim _{s} m_{\theta, e}(s)$ exists,

(P2) if $\gamma_{e}$ and $\delta_{e}$ are total, then additionally $d_{\theta, e} \downarrow$ and we have either $\Gamma_{e}^{V_{e}^{\prime}}\left(z_{\theta, e}\right) \neq$ $m$ or $V_{e}^{\prime} \neq \Delta_{e}^{\left(\theta^{-} m^{\curlyvee} 0^{\omega} \oplus \emptyset^{\prime}\right)\left\lceil d_{\theta, e} .\right.}$

Note that undefined counts as being not equal.

2.3. The construction for $M_{\theta, e}$. Now we give the actions of the module $M_{\theta, e}$.

Step 1: Let $z_{\theta, e}=|\theta|$.

Step 2: Wait for $\gamma_{e}\left(z_{\theta, e}\right) \downarrow$. Using a strong form of the relativized recursion theorem, for each $\sigma \in 2^{\gamma_{e}\left(z_{\theta, e}\right)}$, let $x_{\sigma}>\gamma_{e}\left(z_{\theta, e}\right)$ be a number that we control for $V_{e}^{\prime}$. That is, we may enumerate axioms for $\Phi_{x_{\sigma}}^{X}\left(x_{\sigma}\right)$ and also specify the use of the axioms. In short, we call these axioms for $x_{\sigma}$. Note that $x_{\sigma}$ for different modules are different.

Wait for $\delta_{e}\left(\max _{\sigma} x_{\sigma}\right) \downarrow$. Let $d_{\theta, e}=\delta_{e}\left(\max _{\sigma} x_{\sigma}\right)$, and proceed to Step 3 .

Step 3: We say that $s$ is a recovery stage if $\Gamma_{e}^{Q}\left(z_{\theta, e}\right)[s] \downarrow=m_{\theta, e}[s]$ and

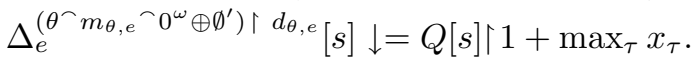

For the clarity of presentation, we assume that the enumeration of $Q_{e}$ is fixed and independent of our actions. In particular we do not follow the customary practice of using a slowdown lemma in the enumeration of the jump. That is, when we define some $\Phi_{x_{\sigma}}^{V_{e}}\left(x_{\sigma}\right)$ to converge, we do not assume that $Q_{e}\left(x_{\sigma}\right)$ responds instantly. If this computation we defined is indeed correct then this will be reflected eventually in $Q_{e}\left(x_{\sigma}\right)$ and we can just wait for it; on the other hand if $V_{e}$ changes before $Q_{e}\left(x_{\sigma}\right)$ responds, then we would have made some progress since the use on the axiom for $x_{\sigma}$ was based on some other "real" computation reflected earlier by $Q_{e}$. Consequently we say that $Q(x)$ is good at stage $s$, if $x$ is an index which we control by the recursion theorem, and $Q_{e}(x)[s]=1$ iff there is a current axiom at $s$ which applies for $x$.

For any set $X \subset 2^{\omega}$ and any number $n \in \omega$, we let $u(n, X)$ denote the (current) use of the computation $\Phi_{n}^{X}(n)$. At each future stage of the construction, for each $\sigma \in 2^{\gamma_{e}\left(z_{\theta, e}\right)}, x_{\sigma}$ will have a mode associated to it, which will be either IN or OUT, reflecting our desire to have $x_{\sigma}$ in or out of $V_{e}^{\prime}$. Initially begin with all $x_{\sigma}$ in mode 
OUT. Unless a change in mode is explicitly stated in the ensuing construction, the mode will not change from one stage to the next.

At all successive stages, $m_{\theta, e}(s)$ outputs the previous value unless $z_{\theta, e}$ is toggled in which case we flip $m_{\theta, e}(s)$.

Stage s: Let $\sigma=Q_{e}\left\lceil\gamma_{e}\left(z_{\theta, e}\right)[s]\right.$.

If $x_{\sigma}$ has mode IN, and there is no axiom that currently applies for $x_{\sigma}$, we enumerate an axiom for $x_{\sigma}$ with use $V_{e, s}\left\lceil\max \left\{u\left(q, V_{e, s}\right) \mid \sigma(q)=1\right\}\right.$.

If $s$ is a recovery stage and $Q_{e}\left(x_{\sigma}\right)$ is good, we call $s$ a good recovery stage and proceed as follows.

Case 1: no axiom for $x_{\sigma}$ applies. Declare $x_{\sigma}$ to have mode IN for stage $s+1$.

Case 2: an axiom for $x_{\sigma}$ applies. Declare $x_{\sigma}$ to have mode OUT for stage $s+1$, and toggle $z_{\theta, e}$.

2.4. Verification. This completes the construction. If $\sigma \subset Q_{e}[s]$ then we will refer to $s$ as a $\sigma$-stage. We first make the following observation.

Lemma 2.1. At all stages $s$ after Step 2 is completed, if an axiom applies for $x_{\tau}$, then it has use $\max \left\{u\left(q, V_{e, s}\right) \mid \tau(q)=1\right\}$ with all the uses defined. Moreover, if $x_{\sigma}$ has mode IN at stage $s$ and $\sigma=Q_{e} \uparrow \gamma_{e}\left(z_{\theta, e}\right)[s]$ then $u\left(q, V_{e, s}\right) \downarrow$ for every $q$ such that $\sigma(q)=1$.

Proof. The first statement follows directly from the second, while the second statement follows from the fact that if $q \in Q_{e}[s]$ then $\Phi_{q}^{V_{e}}(q)[s] \downarrow$.

Lemma 2.2. If $\sigma=Q_{e}\left\lceil\gamma_{e}\left(z_{\theta, e}\right)[s]\right.$ is to the left of $\tau$ and an axiom for $x_{\tau}$ currently applies (with use $u$ ), then $V_{e} \uparrow u$ cannot have been stable since the last $\tau$-stage.

Proof. Since $\tau$ is to the right of $\sigma$ there is a least $q<\gamma_{e}\left(z_{\theta, e}\right)$ such that $\tau(q)=1$ and $\sigma(q)=0$. Since $\sigma(q)=0$, we have $q \notin Q[s]$. We know $V_{e, s}$ extends $\eta$ for some $x_{\tau}$-axiom $\eta$ enumerated earlier (say at $t$ ), hence $\Phi_{q}^{\eta}(q)[t] \downarrow$. This means that $\Phi_{q}^{V_{e}}(q)[s] \downarrow$ which means that $V_{e}$ cannot extend $\eta$ at every stage between the last $\sigma\left\lceil q\right.$-stage and $s$ (otherwise $q \in Q_{e}[s]$ ).

We recall that we only enter Case 1 or 2 at good recovery stages.

Lemma 2.3. If Step 3 is started and $V_{e}$ is $\Delta_{2}^{0}$, then there are only finitely many good recovery stages. Consequently $z_{\theta, e}$ is toggled only finitely often.

Proof. Assume for a contradiction that there are infinitely many good recovery stages. Let $s$ be the stage by which $\emptyset^{\prime}$ has settled on $d_{\theta, e}$. There are at most two possible configurations of $\left(\theta^{\wedge} m_{\theta, e} \frown 0^{\omega} \oplus \emptyset^{\prime}\right)\left\lceil d_{\theta, e}\right.$ after $s$, which differ on the value of $m_{\theta, e}$. Every good recovery stage after $s$ is either a $\sigma_{0}$-stage or a $\sigma_{1}$-stage, where $\sigma_{i}$ corresponds to the configuration with $m_{\theta, e}=i$.

We first claim that there is a stage $s_{1}>s$ such that Case 1 applies. Suppose not. Then at every good recovery stage after $s$, we must have Case 2 applies, whence $z_{\theta, e}$ is toggled. Thus as we visit the good recovery stages after stage $s$, we must be alternating between the two configurations $\sigma_{0}$ and $\sigma_{1}$, in order to recover the toggles. Also, after we have our first good recovery stage with configuration $\sigma_{i}$ after stage $s$, we give $x_{\sigma_{i}}$ mode OUT. Since we are assuming we never enter Case 1 after stage $s$, this means that $x_{\sigma_{i}}$ will remain in mode OUT for the duration of the construction. In particular, it follows that only finitely many axioms are enumerated for $x_{\sigma_{1}}$. Let $t>s$ be a stage where $V_{e}\left\lceil\max \left\{\right.\right.$ of the $x_{\sigma_{1}}$ axioms $\}$ is 
stable. Let $t_{1}>t$ be a good recovery stage with configuration $\sigma_{1}$. Since we were in case 2 , an axiom for $x_{\sigma_{1}}$ applied at stage $t_{1}$. Let $t_{2}>t_{1}$ be a good recovery stage with configuration $\sigma_{0}$. Since $t_{1}>t$, the axiom for $x_{\sigma_{1}}$ still applied at stage $t_{2}$. Now since $\sigma_{0}=Q_{e}\left\lceil\gamma_{e}\left(z_{\theta, e}\right)\left[t_{2}\right]\right.$ is to the left of $\sigma_{1}$, Lemma 2.2 shows that $V_{e}$ could not have been stable on the $x_{\sigma_{1}}$ axiom since the previous $\sigma_{1}$-stage, giving the desired contradiction.

The above contradiction shows that $s_{1}$ exists. Suppose $s_{1}$ is a $\tau$-stage. Let $s_{2}>$ $s_{1}$ be the next good recovery stage (we want to get a contradiction). Since $z_{\theta, e}$ is not toggled by the actions at $s_{1}$, it follows that the configuration of $\left(\theta^{\wedge} m_{\theta, e} 0^{\omega} \oplus \emptyset^{\prime}\right) \uparrow$ $d_{\theta, e}$ at the beginning of $s_{2}$ is the same as at the beginning of $s_{1}$. Hence $s_{2}$ is also a $\tau$-stage. Since $x_{\tau}$ receives mode IN at $s_{1}$, it follows that $x_{\tau}$ has mode IN at the beginning of $s_{2}$, where an axiom for $x_{\tau}$ will be enumerated (if there is not already one). At $s_{1}, Q_{e}\left(x_{\tau}\right)$ must be 0 because of its goodness, which means that at $s_{2}$, $Q_{e}\left(x_{\tau}\right)$ must be again 0 since $s_{2}$ is a recovery stage and a $\tau$-stage. But an axiom for $x_{\tau}$ applies at stage $s_{2}$, and $s_{2}$ is a good stage, so $Q_{e}\left(x_{\tau}\right)=1$, a contradiction.

Lemma 2.4. $M_{\theta, e}$ satisfies (P1) and (P2).

Proof. If $V_{e}$ is $\Delta_{2}^{0}$, then (P1) holds by Lemma 2.3. To show (P2) holds as well we assume that $\delta_{e}, \gamma_{e}$ are total (hence Step 3 is started). Let $\sigma=V_{e}^{\prime}\left\lceil\gamma_{e}\left(z_{\theta, e}\right)\right.$, the true segment of $V_{e}^{\prime}$. Also let $r=V_{e}^{\prime}\left(x_{\sigma}\right)$. Hence there are infinitely many $\sigma$-stages $s$ where $Q_{e}\left(x_{\sigma}\right)[s]=r$. We claim that $Q_{e}\left(x_{\sigma}\right)$ is good at almost every such stage.

For every $p$ such that $\sigma(p)=1, p$ must be in the real $V_{e}^{\prime}$ and so it is easy to see that once $V_{e}$ is stable on these uses, any $x_{\sigma}$-axiom we enumerate applies forever. Hence we only enumerate finitely many axioms for $x_{\sigma}$. If $V_{e}$ extends one of these axioms then at almost every stage $V_{e}[s]$ extends the axiom and also $Q_{e}\left(x_{\sigma}\right)=1$. If $V_{e}$ extends none of these axioms then at almost every $\sigma$-stage where $Q_{e}\left(x_{\sigma}\right)[s]=0$, we have $V_{e}[s]$ extending none of these axioms. Hence $Q_{e}\left(x_{\sigma}\right)$ is good at almost every $\sigma$-stage $s$ where $Q_{e}\left(x_{\sigma}\right)[s]=r$.

Assume for a contradiction that the last condition in (P2) fails. By Lemma 2.3 let $s_{0}$ be a stage by which $\left(\theta^{\frown} m_{\theta, e} 0^{\omega} \oplus \emptyset^{\prime}\right)\left\lceil d_{\theta, e}\right.$ has settled. There are infinitely many stages after $s_{0}$ where $Q_{e}[t] \uparrow 1+\max \left\{x_{\tau}\right\}$ is correct, and each of these is a recovery stage. By the above paragraph we will have infinitely many stages with a long length of agreement, contradicting Lemma 2.3.

We make a further comment. If we further assumed that $\left\{V_{e}\right\}$ is a c.e. approximation for every $e$, then the function $(\theta, e) \mapsto \lim _{s} m_{\theta, e}[s]$ is $\omega+1$-c.e.. To see this, suppose $s$ is a stage where we toggled $z_{\theta, e}$. Follow the proof of Lemma 2.3 and see that after $s$, as long as there is no change to the $\emptyset^{\prime}$ portion of $\left(\theta^{\frown} m_{\theta, e} 0^{\omega} \oplus \emptyset^{\prime}\right)\left\lceil d_{\theta, e}\right.$, we only toggle $z_{\theta, e}$ at most 4 times under Case 2 before Case 1 must apply at a good recovery stage. The second paragraph in the proof of Lemma 2.3 shows that $z_{\theta, e}$ is never toggled again, unless there is a change to the $\emptyset^{\prime}$ portion of $\left(\theta^{\frown} m_{\theta, e} 0^{\omega} \oplus \emptyset^{\prime}\right) \uparrow d_{\theta, e}$. Hence, if $V_{e}$ is c.e., then $z_{\theta, e}$ will be toggled no more than $2 d_{\theta, e}$ many times.

\section{THE FAILURE OF THE ANALOGS OF JUMP INVERSION}

Towards proving our main theorems, the module $M_{\theta, e}$ will meet requirement $R_{e}$, provided that $\theta$ is indeed the initial segment of the characteristic function of $S$. We now show how to combine the modules in such a way that for each $e$, there is a successful $M_{\theta, e}$ module. 
Given a sequence $\left\{V_{e}\right\}$, we apply the previous section to get $m_{\theta, e}, d_{\theta, e}$. We now specify an approximation $S[s]$ by the following. First we order the finite binary strings by the following: $\lambda \prec 1 \prec 0 \prec 11 \prec 10 \prec 01 \prec 00 \prec 111 \prec \cdots$. Hence $\prec$ refers to the ordering obtained by first considering increasing length, and then reverse lexicographic ordering. For each $\eta$ we will have an associated binary string $\theta_{\eta}$, and the corresponding $z_{\theta_{\eta},|\eta|}$ as defined in module $M_{\theta_{\eta},|\eta|}$. That is, $z_{\theta_{\eta},|\eta|}=\left|\theta_{\eta}\right|$. For convenience, we will let $z_{\eta}$ denote $z_{\theta_{\eta},|\eta|}$. We will arrange it so that for $\eta^{\prime} \prec \eta$, we have $z_{\eta^{\prime}}<z_{\eta}$. Basically $z_{\eta}$ serves as a pointer, and points to a location of $S$ where $S\left(z_{\eta}\right)[s]$ will be approximated by the digits of $m_{\theta_{\eta},|\eta|}$. Each $\eta$ codes a guess as to the membership of $z_{\eta^{\prime}}$ in $S$ for $\eta^{\prime} \prec \eta$. We will have $\theta_{\eta}$ represent the $\eta$-guess as to the correct initial segment $S \uparrow\left|\theta_{\eta}\right|$. As we give the stage by stage construction of $S$, we will move the pointers $z_{\eta}$, but each $z_{\eta}$ will only be moved finitely often. Although $z_{\eta}$ is defined to be the length of $\theta_{\eta}$, in practice we will define $z_{\eta}$ first, and later define $\theta_{\eta}$. At every stage $s$, if $y$ is not being pointed at (i.e. $y \neq z_{\eta}$ for any $\eta)$, then we will have $S(y)[s]=0$.

We give a few notations to be used. Define $T_{s}$ to be a string of finite length, which can be thought of as the current approximation to the "true strategies". Loosely speaking, only those $z_{\eta}$ where strategy $\eta \subset T$ will be the important ones; the other $z_{\eta}$ with $\eta$ not on $T$ are just red herrings; they are the artifacts produced by our wrong guesses. $T_{s}$ is defined inductively by: $T_{s}(n)=S\left(z_{T_{s} \uparrow n}\right)[s]$. Proceed this way until we hit the first undefined $z_{-}$.

At stage $s$ to read the next digit of $m_{\theta, e}$ means to do the obvious thing: if this is the first time we encounter this instruction then we output $m_{\theta, e}(0)$. Otherwise output $m_{\theta, e}(k+1)$ where $m_{\theta, e}(k)$ was the previous digit read by the construction.

Construction of $S$ : at $s=0$ make every $z_{\eta}, \theta_{\eta}$ undefined. At stage $s>0$, only finitely many $z_{\eta}, \theta_{\eta}$ have been defined at the end of stage $s-1$. Go through all such $\eta$ in increasing order, and for each we (inductively) update $\theta_{\eta}$ and specify $S\left(z_{\eta}\right)[s]$. For $z_{\lambda}$ we let $\theta_{\lambda}=\lambda$ and set $S\left(z_{\lambda}\right)[s]=$ the next digit of $m_{\theta_{\lambda}, 0}$.

Now assume that $S\left(z_{\eta^{\prime}}\right)[s]$ has been defined for all $\eta^{\prime}<\eta$. We define $\theta_{\eta}$ as follows. For $y<z_{\eta}$ such that $y \neq z_{\eta^{\prime}}$ for any $\eta^{\prime}$, set $\theta_{\eta}(y)=0$. If $y<z_{\eta}$ is such that $y=\eta^{\prime}$, then necessarily $\eta^{\prime} \prec \eta$. If $\eta^{\prime}$ is lexicographically to the right of $\eta \uparrow\left|\eta^{\prime}\right|$ then set $\theta_{\eta}\left(z_{\eta^{\prime}}\right)=0$. If $\eta^{\prime}$ is left of $\eta \uparrow\left|\eta^{\prime}\right|$ then set $\theta_{\eta}\left(z_{\eta^{\prime}}\right)=S\left(z_{\eta^{\prime}}\right)[s]$. Otherwise $\eta^{\prime}=\eta \uparrow\left|\eta^{\prime}\right|$ and we let $\theta_{\eta}\left(z_{\eta^{\prime}}\right)=\eta\left(\left|\eta^{\prime}\right|\right)$. Next we define $S\left(z_{\eta}\right)[s]$ by the following. Note first of all that $T_{s} \uparrow|\eta|$ can be evaluated at this point. If $T_{s} \uparrow|\eta|=\eta$ then we let $S\left(z_{\eta}\right)[s]$ be the next digit of $m_{\theta_{\eta},|\eta|}$. If $T_{s} \uparrow|\eta|$ is left of $\eta$ then let $S\left(z_{\eta}\right)[s]=0$. Otherwise if $T_{s}\left\lceil|\eta|\right.$ is right of $\eta$ we let $S\left(z_{\eta}\right)[s]=S\left(z_{\eta}\right)[s-1]$.

If some $d_{\theta_{\eta},|\eta|}$ has converged at stage $s$, we make all $z_{\eta^{\prime}}, \theta_{\eta^{\prime}}$ undefined for all $\eta^{\prime}>\eta$ and go to the next stage. Otherwise the above stops naturally when we find some least $\eta$ with $z_{\eta}$ not defined at stage $s-1$. We then pick a fresh value for $z_{\eta}$ and set $S\left(z_{\eta}\right)[s]=0$.

Finally let $S(x)=\liminf _{s} S(x)[s]$. It is clear that $z_{\eta}$ eventually settles on a final value for each $\eta$, and also that $\left|T_{s}\right| \rightarrow \infty$. Let $T$ be the leftmost path specified infinitely often by $T_{s}$. We first show that $T$ actually reflects the correct $\eta$ 's:

Lemma 3.1. For every $\eta \subset T$, we have $\theta_{\eta}$ eventually settles, $\theta_{\eta} \subset S$ and $S\left(z_{\eta}\right)=$ $T(|\eta|)=\liminf m_{\theta_{\eta},|\eta|}$.

Proof. We proceed inductively on $|\eta|$. The statement clearly holds if $|\eta|=0$ so take $|\eta|>0$. After $z_{\eta}$ settles, the value of $\theta_{\eta}$ and also $S\left\lceil z_{\eta}\right.$ will be decided on the places $\left\{z_{\eta^{\prime}} \mid \eta^{\prime} \prec \eta\right\}$. There are three cases. If $\eta^{\prime}$ is right of $\eta \uparrow\left|\eta^{\prime}\right|$ then $\theta_{\eta}\left(z_{\eta^{\prime}}\right)$ 
is always 0 , while at infinitely many stages $s, T_{s} \supset \eta$ which makes $S\left(z_{\eta^{\prime}}\right)[s]=0$ infinitely often. If $\eta^{\prime}$ is left of $\eta \uparrow\left|\eta^{\prime}\right|$ then $T_{s}$ is right of $\eta^{\prime}$ at every stage after some $s_{0}$. Hence $S\left(z_{\eta^{\prime}}\right)[s]=S\left(z_{\eta^{\prime}}\right)\left[s_{0}\right]$ for all $s>s_{0}$ and also $\theta_{\eta}\left(z_{\eta^{\prime}}\right)$ will agree with $S\left(z_{\eta^{\prime}}\right)\left[s_{0}\right]$. Finally if $\eta^{\prime} \subset \eta$ then inductively let $\theta_{\eta^{\prime}}$ be the limit. It is easy to see that the value of $\theta_{\eta}\left(z_{\eta^{\prime}}\right)=\eta\left(\left|\eta^{\prime}\right|\right)=T\left(\left|\eta^{\prime}\right|\right)=S\left(z_{\eta^{\prime}}\right)$. Hence $\theta_{\eta}$ eventually settles and $\theta_{\eta} \subset S$.

Since $\eta$ is on $T$, hence for almost all $s$ we have $T_{s}$ is right of $\eta$ (where $S\left(z_{\eta}\right)[s]$ is unchanged from the previous stage) or $T_{s} \supset \eta$ (in which the next digit of $m_{\theta_{\eta},|\eta|}$ is $\operatorname{read})$. Hence $S\left(z_{\eta}\right)=\lim \inf m_{\theta_{\eta},|\eta|}$. To see that this value is the same as $T(|\eta|)$, observe that $T(|\eta|)=\liminf \left\{T_{s}(|\eta|) \mid T_{s} \supset \eta\right\}=\liminf \left\{S\left(z_{\eta}\right)[s] \mid T_{s} \supset \eta\right\}=$ $\lim \inf m_{\theta_{\eta}, \eta \mid}$.

Lemma 3.2. For every $e$, if $V_{e}$ is $\Delta_{2}^{0}$, then $V_{e}^{\prime} \not \equiv{ }_{w t t} S \oplus \emptyset^{\prime}$.

Proof. WLOG we assume that $V_{e}^{\prime}=\Delta_{e}^{S \oplus \emptyset^{\prime}}$ and $S=\Gamma_{e}^{V_{e}^{\prime}}$ with use bounded by $\delta_{e}, \gamma_{e}$ (which are total). Let $\eta=T \uparrow e$. By Lemmas 2.4 and $3.1 S\left(z_{\eta}\right)=\lim m_{\theta_{\eta}, e}$ where $\theta_{\eta} \subset S$. Since $d_{\theta_{\eta}, e} \downarrow$ then the initialization in the construction of $S$ ensures that in fact $\left(S \oplus \emptyset^{\prime}\right) \mid d_{\theta_{\eta}, e}=\left(\theta_{\eta} \frown \lim m^{\frown} 0^{\omega} \oplus \emptyset^{\prime}\right)\left\lceil d_{\theta_{\eta}, e}\right.$. A contradiction to the last condition of (P2) follows.

We now obtain as corollaries, the following three statements.

Theorem 3.3. For any computable sequence of $\Delta_{2}^{0}$ sets $\left\{V_{e}\right\}_{e \in \mathbb{N}}$ (given by their $\Delta_{2}^{0}$ indices), there exists a $\Delta_{2}^{0}$ set $S \geq_{t t} \emptyset^{\prime}$ such that for every $e, V_{e}^{\prime} \not \equiv w_{w t} S$.

Proof. Apply the results of the past two sections, and $S \oplus \emptyset^{\prime}$ is the desired set. Note that $S$ is $\Delta_{2}^{0}$ because of $(\mathrm{P} 2)$ and the fact that it is easy to prove that $\left\{T_{s}\right\}$ itself is a $\Delta_{2}^{0}$ approximation.

Theorem 3.4. There exists an $\omega+1$-c.e. set $S>_{t t} \emptyset^{\prime}$ such that there is no c.e. set $A$ with $A^{\prime} \equiv{ }_{w t t} S$.

Proof. Theorem 3.3 gives a us a $\Delta_{2}^{0}$ set $S$. To see that $S$ can be made $\omega+1$-c.e., use the fact that every module will reach a limit and modify the construction of $S$ slightly to ensure that each time $S\left\lceil z_{\eta}[s]\right.$ changes we also reset $z_{\eta}$. It is not hard to see that the ensuing approximation for $S$ will be a $\omega+1$-c.e.. We sketch the reason why, and leave the details to the reader. The value $S\left(z_{\eta}\right)[s]$ depends directly on the value of $T_{s} \uparrow|\eta|$, which in turn depend on $S\left(z_{\nu}\right)[s]$ where $\nu<\eta$. As long as $S\left\lceil z_{\eta}[s]\right.$ remains unchanged, we will either output 0 for $S\left(z_{\eta}\right)[s]$, or the digits of $m_{\theta_{\eta},|\eta|} \cdot \theta_{\eta}$ will also not change as long as $S\left\lceil z_{\eta}[s]\right.$ remains fixed. Hence the number of changes in $S\left(z_{\eta}\right)$ is at most the number of flips in $m_{\theta_{\eta},|\eta|}$ (until $z_{\eta}$ is cancelled). This number can be computed by the comments after Lemma 2.4 .

Theorem 3.5. There exists a $\Sigma_{2}^{0}$ set $S>_{t t} \emptyset^{\prime}$ such that there is no $\Delta_{2}^{0}$ set $A$ with $A^{\prime} \equiv{ }_{w t t} S$.

Proof. Use a list of all possible $\Delta_{2}^{0}$ indices.

\section{REFERENCES}

[And08] B. Anderson. Automorhisms of the truth-table degrees are fixed on some cone. 2008. Preprint.

[Coo04] S. Barry Cooper. Computability theory. Chapman \& Hall/CRC, Boca Raton, FL, 2004. 
[Dem88] O. Demuth. Remarks on the structure of tt-degrees based on constructive measure theory. Commentationes Mathematicae Universitatis Carolinae, 29(2):233-247, 1988.

[DR89] R. Downey and J. Remmel. Classification of degree classes associated with r.e. subspaces. Annals of Pure and Applied Logic, 42:105-124, 1989.

[Fri58] R. Friedberg. A criterion for completeness of degrees of unsolvability. Journal of Symbolic Logic, 22:159-160, 1958

[Moh84] J. Mohrherr. Density of a final segment of the truth-table degrees. Pacific J. Math, 115:409-419, 1984.

[Pos44] E. Post. Recursively enumerable sets of positive integers and their decision problems. Bulletin of the American Mathematical Society, 50:284-316, 1944.

[Rob71] R. Robinson. Jump restricted interpolation in the recursively enumerable degrees. Annals of Mathematics, 93 (2):586-596, 1971.

[RS08a] J. Reimann and T. Slaman. Measures and their random reals. 2008. Preprint.

[RS08b] J. Reimann and T. Slaman. Probability measures and effective randomness. 2008. Preprint.

[Sac63] G. Sacks. Recursive enumerability and the jump operator, volume 108. 1963.

[Sho59] J. Shoenfield. On degrees of unsolvability. Annals of Mathematics, 69:644-653, 1959.

[Soa87] R. Soare. Recursively enumerable sets and degrees. Perspectives in Mathematical Logic. Springer-Verlag, 1987.

Department of Pure Mathematics, University of Waterloo, Waterloo, On, Canada N2L 3G1

$U R L$ : www.math. uwaterloo.ca/ csima

E-mail address: csima@math.uwaterloo.ca

School of Mathematics, Statistics and Computer Science, Victoria University of Wellington, PO Box 600, Wellington, New Zealand

E-mail address: Rod.Downey@ecs.vuw.ac.nz

School of Mathematics, Statistics and Computer Science, Victoria University of Wellington, PO Box 600, Wellington, New Zealand

E-mail address: Keng. Meng.Ng@ecs.vuw.ac.nz 